\title{
THE CORRESPONDENCE BETWEEN PHONETIC TRANSCRIPTION AND ORTHOGRAPHIC TRANSCRIPTION IN ENGLISH
}

\author{
By: \\ Surono \\ Lecturer of University of Ahmad Dahlan Yogyakarta \\ Doctoral Students of Linguistics Department, \\ Postgraduate Program, Sebelas Maret University \\ Email: Ling_sure@yahoo.com
}

\begin{abstract}
This study entitled The Correspondence between Phonetic Transcription and Orthographic Transcription in English is intended to describe how phonetic symbols correspond to orthographic symbols (alphabets), and to find the most complex and simplest phonetic symbols and orthographic symbols (alphabets). This study belongs to descriptive qualitative type. The research object is the phonetic transcription and orthographic transcription. The data were in the forms of words and their phonetic transcription and they were gathered from two dictionaries, namely Oxford Advanced Learner's Dictionary of Current English written by Hornby (1986) in complete version and Longman Dictionary of Contemporary English written by Longman (2001). The data were gathered by observation method followed by noting technique. Then, dividing-key-factor technique using mental ability in dividing the data was used to analyze the data. The research findings show that in English the phonetic symbols (transcription) have one-to-many correspondence with the orthographic symbols (transcription) viewed from both sides. Viewed from the phonetic symbols, there are only two symbols which have one-to-onecorrespondence, namely [æ] and [כ], but viewed from the orthographic symbols, all alphabets have one-to-manycorrespondence. The most complex phonetic symbol referring to vowels is [i:], while the simplest one is [æ]. The most complex phonetic symbols referring to diphthongs are [iə] and [uə], while the simplest one is [au]. Finally, the most complex orthographic symbol (alphabet indicating vowels) is $\boldsymbol{o}$, while the simplest one is $\boldsymbol{e}$.
\end{abstract}

Key words: phonetic transcription, orthographic transcription, one-to-many correspondence, one-to-one correspondence

\section{INTRODUCTION}

One of the characteristics of language is that it is unique. Each language in the world has their own uniqueness or specialties. The uniqueness may cover phonological, 
morphological, syntactic, or even semantic aspects. The phonological aspect is the most complex one because it is related to the language sounds (spoken language) which may lead to the uncontrolled variability.

Phonology is the study of the speech sounds and the ways how they are produced and used to convey meanings. The object of analysis in phonology is speech sounds or phonemes (see Sloat et al., 1978). Phoneme is the smallest distinctive unit of sounds of a language which can distinguish meanings (Mcmahon, 2002; Verhaar, 1996; Chaer, 1994; Hyman, 1975).

Accoding to Widdowson (1995:4), each language has finite phonological systems by which the speakers communicate. It means to show the sound change in a word (orthographic transcription) can be done by changing or replacing a given phoneme (phonetic transcription) with another phoneme. It implies that the ways (systems) for pronouncing individual letters (alphabets) are different if they are used in words.

In principle, language is firstly spoken, written language is secondary. Trask (in Widdowson, 1995: 4) explains "since all languages are spoken, they must have phonetic and phonological system. Since they all have words and sentences, they must also have morphology and syntax." Of course, the systems of spoken language and written language are different, but they can be related. Written language exists due to (based on) the spoken language. Both have different symbolization. Spoken language is symbolized with phonetic symbols (transcription), whereas written language is symbolized with letters or alphabets (orthographic transcription). The phonetic symbols are based on the symbols standardized by International Phonetic Association (IPA), whereas the orthographic symbols are universally based on the alphabets used by most languages in the world.

The phonetic transcription is used to guide and control the pronunciation system in oral communication using phonetic symbols, whereas orthographic transcription is used to guide and control the writing system in written communication using the orthograpic symbols called alphabets. The alphabets have their own pronunciation which is mostly different from the symbols used in phonetic transcription.

English has a very complex system of sound especially when it is related to the orthographic system. The sounds of combined phonemes in words are highly different from that of individual phonemes representaing letters. As an illustration, the following example can be considered: (a) the alphabet a in English can correspond to the phonetic symbols in 
various ways, e.g. [ a: ] in father, [ ei ] in bass, [æ ] in man, and [ $\partial$ ] in above; (b) in contrast, the sound [ $\mathrm{k}$ ] can correspond to several alphabets, e.g. $\boldsymbol{k i n k e y , ~} \boldsymbol{c}$ in $\boldsymbol{c a n}$, $\boldsymbol{c h}$ and $\boldsymbol{q}$ in technique.

There are many problems related to the spoken and written language which can be analyzed, e.g. the symbolization or transcription, the function, the production and the writing system, etc. In this research, the problem which is analyzed is limited to the symbolization system particularly the correspondence between the phonetic transcription and the orthographic transcription. The phonetic transcription refers to the vowel and diphthong symbols and the orthographic transcription refers to the alphabets indicating vowels, namely $A, E, I, O$, and $U$.

Vowels and diphthongs are two kinds of sounds which are principally produced in the same manner. Diphthongs are produced similarly to vowels. Ramelan (1985:48) explains that "vowel is a voiced sound during the production of which the air passes out freely and continuously throughout the middle of the mouth without such narrowing as would cause any audible friction".

To guide the research to the focused problems and to get appropriate and accurate solution, the following are the statements of the problems to be solved: (1) How is the correspondence between the phonetic transcripton and the orthographic transcription in English?; (2) Which English phonetic symbols are the most complex and the simplest?; (3) Which English alphabets are the most complex and the simplest?

\section{METHODS}

This research belongs to descriptive qualitative because this is intended to describe the observable phenomena through the data analysis to solve the problems determined before hand (Djajasudarma, 1993: 9). The data which were analyzed here took the nonnumerical forms, namely the words and their phonetic transcription containing vowels and the alphabets indicating vowels.

The objects of this research are the phonetic transcription and the orthographic transcription both of which can be found in English monolingual dictionaries. So, in this case, thoses dictionaries serve as the sources of the data. Two dictionaries were used to get the data in the hope that variations of the correspondence between the two kinds of transcription could be recognized. They were Oxford Advanced Learner's Dictionary of Current English written 
by Hornby (1986) in complete version andLongman Dictionary of Contemporary English written by Longman (2001).The phonetic transcription and the orthographic transcription were compared in terms of the existing vowels and diphthongs and the alphabets indicating vowels.

In this research, the resercher was the key instrument to gather the data. Djajasudarma (1993:11) states that in qualitative research the researcher is the primary data gathering instrument. In this case, the researcher observed the English monolingual dictionaries to gaather the suitable data. That is why the method of gathering the data used in this research was observation method. This was applied by observing the words and their phonetic transcription found in the dictionaries.

The the choice of technique and procedures for gathering the data is influenced by many factors related to the relevant disciplines (Seliger and Shohamy, 1989:37). Based on Sudaryanto's (1993) opinion, the technique for gathering the data used in this research was noting technique, that is, the data gathered from the dictionaries were noted in data cards prepared previously(see also Singleton and Straits, 1999: 456-464).

The typology of the data was made based on the distribution of the phonetic symbols (vowel sounds) and the alphabets indicating vowels. The distributions were in initial, mid, and final positions of English words.

Meanwhile, the technique of data analysis used was dividing-key-factor technique (Sudaryanto, 1993: 21)using mental ability in dividing the data. The data analysis was done inductively (Djajasudarma, 1993:13) because this technique can reveal the complex phenomena containing in the data. (Cf. Singleton and Straits, 1999: 41).

\section{FINDING AND DISCUSSION}

In this part, the researcher will show the research findings and the discussion integratedly. The research findings are showed based on the research problems stated in partI.

\section{The Correspondence between Orthographic Transcription and Phonetic Transcription}

a. From Orthographic Writing (Alphabets) to Phonetic Writing

Concerning the alphabets indicating the vowels, they are more complex and complicated than those indicating the consonants. Because this kind of alphabets corresponds to the vowels which are also complex and complicated, those alphabets show many 
variations. The following table indicates the correspondence between the vowels (phonetic transcription) and the alphabets indicating the vowels (orthographic transcription).

Tabel 1

The Correspondence from the Orthographic Transcription (Alphabets Indicating the Vowels) to the Phonetic Transcription (Vowel Symbols)

\begin{tabular}{|c|c|c|c|c|c|c|c|c|}
\hline \multirow{3}{*}{ No } & \multirow{3}{*}{$\begin{array}{l}\frac{n}{0} \\
\frac{0}{\pi} \\
\frac{\pi}{2} \\
\frac{1}{2}\end{array}$} & \multirow{3}{*}{ 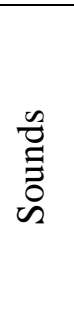 } & \multicolumn{6}{|c|}{ 3.1 D I S T R I B U T I O N } \\
\hline & & & \multicolumn{2}{|c|}{ Initial Position } & \multicolumn{2}{|c|}{ Mid Position } & \multicolumn{2}{|c|}{ Final Position } \\
\hline & & & $\begin{array}{c}\text { Words and } \\
\text { page }\end{array}$ & $\begin{array}{l}\text { Phonetic } \\
\text { transcript } \\
\text { ion }\end{array}$ & $\begin{array}{c}\text { Words and } \\
\text { page }\end{array}$ & $\begin{array}{c}\text { Phonetic } \\
\text { transcript } \\
\text { ion }\end{array}$ & $\begin{array}{l}\text { Words } \\
\text { and } \\
\text { page }\end{array}$ & $\begin{array}{c}\text { Phonetic } \\
\text { transcript } \\
\text { ion }\end{array}$ \\
\hline \multirow[t]{12}{*}{1} & \multirow[t]{12}{*}{$\mathrm{A}$} & [æ] & Avenue/70 & [ævəјu] & $\begin{array}{l}\text { Balance/2 } \\
6\end{array}$ & [bæləns] & - & - \\
\hline & & & Access $/ 3$ & [æks\&s] & Land/719 & [lænd] & - & - \\
\hline & & [a:] & Ask/59 & [a:sk] & Half/578 & [ha:f] & $\begin{array}{l}\text { Gaga/4 } \\
84\end{array}$ & [ga:ga:] \\
\hline & & & Aunt/68 & [a:nt] & $\begin{array}{l}\text { Garden/52 } \\
9\end{array}$ & [ga:dn] & $\mathrm{Pa} / 909$ & [pa:] \\
\hline & & $\begin{array}{c}\text { [J: } \\
\text { ] }\end{array}$ & Also/25 & [כ:1səu] & Bald/29 & [bJ:ld] & - & - \\
\hline & & & Alright/25 & [כ:lrait] & Walk/189 & [w]:k] & - & - \\
\hline & & [ə] & Aloud/12 & [əlaud] & Salary/370 & [sælərI] & $\begin{array}{l}\text { Camera } \\
/ 168\end{array}$ & $\begin{array}{l}\text { [kæmərə } \\
\text { ] }\end{array}$ \\
\hline & & & About $/ 3$ & [obaut] & $\begin{array}{l}\text { Balance/5 } \\
9\end{array}$ & [bæləns] & $\begin{array}{l}\text { Cinema } \\
/ 209\end{array}$ & [sinəmə] \\
\hline & & {$[\varepsilon]$} & Any/24 & [Eni] & Canary/59 & [kənerI] & - & - \\
\hline & & & $\begin{array}{l}\text { Anyone/2 } \\
4\end{array}$ & [Eniw $\Lambda \mathrm{n}]$ & - & - & - & - \\
\hline & & [ei] & Able/45 & [eibl] & Cake/58 & [keik] & - & - \\
\hline & & & Ace $/ 10$ & [eis] & Fable/448 & [feibl] & - & - \\
\hline \multirow[t]{4}{*}{2} & \multirow[t]{4}{*}{$\mathrm{E}$} & {$[\varepsilon]$} & Edit/401 & [Edit] & Cell/187 & [scl] & - & - \\
\hline & & & Elbow/704 & [Elbəu] & $\begin{array}{l}\text { Possess/31 } \\
9\end{array}$ & [pəzes] & - & - \\
\hline & & [i:] & Eel/131 & [i:1] & $\begin{array}{l}\text { Exceed/14 } \\
1\end{array}$ & [Iksi:d] & $\begin{array}{l}\text { Coffee/ } \\
229\end{array}$ & [kJfi:] \\
\hline & & & Equal/289 & [i:kwəl] & Cedar/187 & [si:də:] & $\begin{array}{l}\text { Flee/48 } \\
7\end{array}$ & [fli:] \\
\hline
\end{tabular}




\begin{tabular}{|c|c|c|c|c|c|c|c|c|}
\hline & & [ï] & Era/289 & [iərə] & Here/60 & [hiə] & - & - \\
\hline & & & - & - & Mere/531 & [miə] & - & - \\
\hline 3 & \multirow[t]{8}{*}{ I } & {$[\mathrm{I}]$} & $\begin{array}{l}\text { Import/33 } \\
8\end{array}$ & [ImpJ:t] & Big/108 & [bIg] & $\begin{array}{l}\text { Brocoli } \\
\text { /81 }\end{array}$ & [brJkəlI] \\
\hline & & & Idiom/630 & [IdIəm] & $\mathrm{Kid} / 369$ & [kId] & $\begin{array}{l}\text { confetti } \\
/ 87\end{array}$ & [kənf\&tI] \\
\hline & & [i:] & - & - & Brief/79 & [bri:f] & - & - \\
\hline & & & - & - & Cheif/109 & [tfi:f] & - & - \\
\hline & & [ə:] & - & - & Dirty/184 & [də:tI] & - & - \\
\hline & & & - & - & Third/694 & [Oə:d] & - & - \\
\hline & & [ai] & Idea/588 & [aidiə] & Arise/18 & [əraiz] & $\begin{array}{l}\text { Alibi/2 } \\
8\end{array}$ & [æləbai] \\
\hline & & & Ice/587 & [ais] & Child/192 & [taild] & $\begin{array}{l}\text { Alkali/ } \\
29\end{array}$ & [ælkəlai] \\
\hline \multirow[t]{14}{*}{4} & \multirow[t]{14}{*}{$\mathrm{O}$} & [כ] & $\begin{array}{l}\text { Object/45 } \\
6\end{array}$ & [Jbjek] & Cloth/119 & {$[\mathrm{kl} \partial \theta]$} & - & - \\
\hline & & & Office/803 & [JfIs] & $\begin{array}{l}\text { Choice/11 } \\
1\end{array}$ & [tOis] & - & - \\
\hline & & [J: & Oral/815 & [J:rəl] & Board/119 & [bJ:(r)d] & - & - \\
\hline & & & $\begin{array}{l}\text { Orchestra/ } \\
815\end{array}$ & [O:kIstrə] & $\begin{array}{l}\text { Bought/12 } \\
8\end{array}$ & [bJ:t] & - & - \\
\hline & & [u:] & Ooze/990 & [u:z] & $\begin{array}{l}\text { Bloom/11 } \\
6\end{array}$ & [blu:m] & $\begin{array}{l}\text { Zoo/18 } \\
92\end{array}$ & [zu:] \\
\hline & & & $\begin{array}{l}\text { Oodles/99 } \\
0\end{array}$ & [u:dlz] & Boom/125 & [bu:m] & $\begin{array}{l}\text { Too/12 } \\
60\end{array}$ & [tu:] \\
\hline & & {$[\mathrm{U}]$} & Oops/990 & [Ups] & Book/124 & [bUk] & - & - \\
\hline & & & $\begin{array}{l}\text { Oomph/99 } \\
0\end{array}$ & [Umf] & Good/512 & [gUd] & - & - \\
\hline & & $\begin{array}{c}{[\partial u} \\
]\end{array}$ & Oak/973 & [ouk] & $\begin{array}{l}\text { Borrow/14 } \\
1\end{array}$ & [bJrəu] & Go/604 & [gəu] \\
\hline & & & Oath/973 & [əuð] & Bow/146 & [bəu] & $\begin{array}{l}\text { So/136 } \\
3\end{array}$ & [səu] \\
\hline & & $\begin{array}{c}\text { [uə } \\
\text { ] }\end{array}$ & - & - & Poor/896 & [puə] & - & - \\
\hline & & & - & - & Tour/1530 & [tuə] & - & - \\
\hline & & {$[\Lambda]$} & Other/819 & {$[\Lambda$ ðə(r)] } & Come/223 & {$[\mathrm{k} \Lambda \mathrm{m}]$} & - & - \\
\hline & & & Onio/809 & [ $\Lambda$ niən] & Flood/449 & {$[\mathrm{fl} \Lambda \mathrm{d}]$} & - & - \\
\hline
\end{tabular}




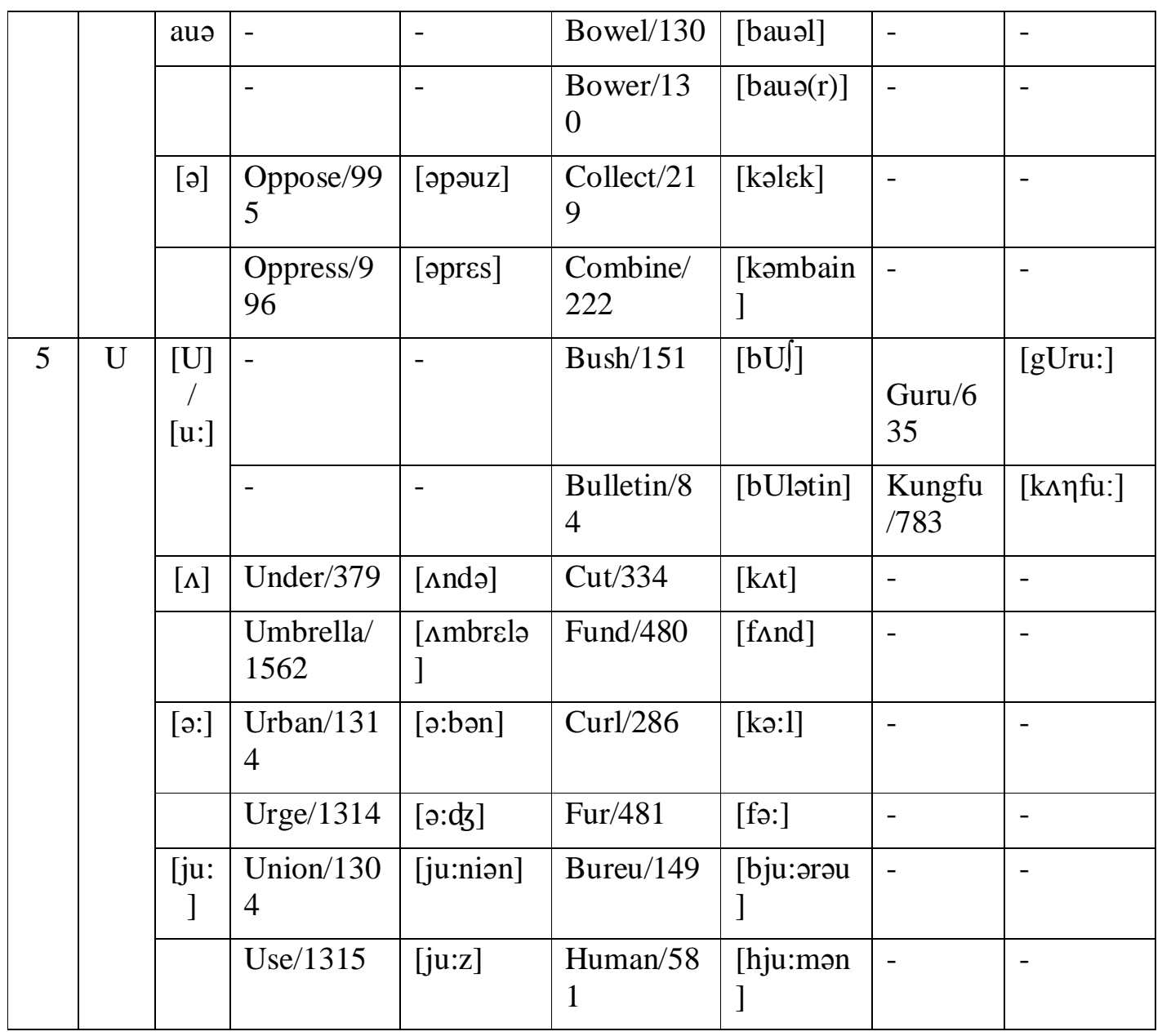

The correspondence between the orthographic transcription (alphabets indicating vowels) and the phonetic transcription (vowels) is more complex than between the orthographic transcription (alphabets indicating consonants) and the phonetic transcription. All the alphabets indicating vowels correspond to the phonetic transcription in many forms, namely $1: 3-9$ with their own contexts.

It can be seen from table 1 that the alphabets Eand $I$ have simpler correspondence than the other three: $A, U, O$. The alphabet $E$ has three forms of correspondence, namely $[\varepsilon]$, [i:], and [iə]. The vowel [E] and the diphthong [iə] correspond to $E$ in initial and mid positions of the words; but the [iə] in initial position is rarely found. Meanwhile, the vowel [i:] for $E$ can be found in all positions. Then, the alphabet $I$ has four forms of correspondence, namely [I], [i:], [ə:], and [ai]. The vowel [I] and the diphthong [ai] for Ican be found in all positions; while if $I$ is in mid position, it corresponds to [i:] or [ə:] in different contexts. 
Another alphabet, $U$, has five forms of correspondence, namely [U], [u:], [ $\Lambda$ ], [ə:], and [ju:]. The vowel sound [u:] for $U$ occurs at final position, while $E$ in mid position corresponds to $[\mathrm{U}],[\Lambda]$, [ə:], and [ju:]. The last three sounds can also correspond to $E$ in initial position. The more forms of correspondence can be seen in the alphabet $A$, that is, it has six forms, namely [æ], [a:], [כ:], [ə], [ع], and [ei]. All these forms correspond to the alphabet $A$ in initial and mid positions. However, the correspondence between $A$ and $[\varepsilon]$ occurs rarely in mid position. Then, when $A$ is at final position it corresponds to [a:] and [ə].

The alphabet $O$ has a correspondence with the phonetic transcription in most forms,

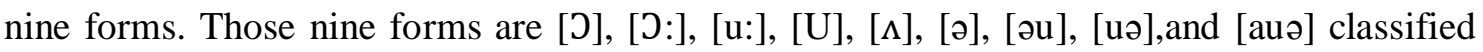
into monophthong, diphthong, and triphthong. The $O$ which corresponds to triphthong is a special case, meaning that it occurs in a very special context. The alphabet $O$ which corresponds to [uə] and [auə] usually appears in mid position, while the other sounds for $O$ can be found in initial and mid positions, except the sounds [u:] and [ou] which can also be found for $O$ in final position.

\section{b. From Phonetic Transcription (Symbols) to Orthographic Transcription}

The discussion on the correspondence between the phonetic transcription and orthographic transcription seen from phonetic transcription (vowels and diphthongs) is divided into some parts presented in different tables. This view is taken in order to clearly know how a vowel or diphthong corresponds to the alphabets.

Viewed in terms of their correspondence with the orthographic transcription, vowels, including diphthongs, show a more complex phenomenon than consonants. It is only the vowels [æ] (table 2) and [כ] (table 3) which have one-to-one correspondence with the orthographic transcription, although their distribution is not complete. The vowel [æ] has a consistent correspondence with the alphabet $\boldsymbol{a}$ wherever it is. The same is true for the alphabet $\boldsymbol{o}$ which consistently corresponds to the vowel [כ] either in initial, mid, or final position of a word. Meanwhile, the vowel [a:] which seems to be simple corresponds to the alphabets $\boldsymbol{a}$ and $\boldsymbol{a} \boldsymbol{r}$ in all positions. It can be seen in the following table. 
Tabel 2

Correspondence from Phonetic Transcription (Front and Central Vowels) to the Orthographic Transcription (Alphabets)

\begin{tabular}{|c|c|c|c|c|c|c|c|c|}
\hline \multirow{3}{*}{ No } & \multirow{3}{*}{$\begin{array}{l}\tilde{z} \\
\Xi \\
\tilde{\Xi} \\
\tilde{s}\end{array}$} & \multirow{3}{*}{$\begin{array}{l}\frac{n}{0} \\
\frac{0}{0} \\
\frac{\pi}{2} \\
\frac{2}{4}\end{array}$} & \multicolumn{6}{|c|}{3.2 D I S T R I B U T I O N } \\
\hline & & & \multicolumn{2}{|c|}{ Initial Position } & \multicolumn{2}{|c|}{ Mid Position } & \multicolumn{2}{|c|}{ Final Position } \\
\hline & & & $\begin{array}{c}\text { Words and } \\
\text { page }\end{array}$ & $\begin{array}{l}\text { Phonetic } \\
\text { transcript } \\
\text { ion }\end{array}$ & $\begin{array}{c}\text { Words and } \\
\text { page }\end{array}$ & $\begin{array}{c}\text { Phoneti } \\
\text { c } \\
\text { transcri } \\
\text { ption }\end{array}$ & $\begin{array}{l}\text { Words } \\
\text { and page }\end{array}$ & $\begin{array}{l}\text { Phonetic } \\
\text { transcript } \\
\text { ion }\end{array}$ \\
\hline \multirow[t]{14}{*}{1} & \multirow{14}{*}{$\begin{array}{c}\text { [ i: } \\
\text { ] }\end{array}$} & ee & $\mathrm{Eel} / 369$ & [i:1] & Creek/97 & [kri:k] & Free/163 & [fri:] \\
\hline & & & - & - & Green/180 & [gri:n] & Knee/20 & [ni:] \\
\hline & & $\begin{array}{c}\mathrm{ie} / \mathrm{e} \\
\mathrm{i}\end{array}$ & - & - & Brief/47 & [bri:f] & $\begin{array}{l}\text { Calorie/5 } \\
9\end{array}$ & [kæləri:] \\
\hline & & & - & - & Thief/451 & {$[\theta \mathrm{i}: \mathrm{f}]$} & $\begin{array}{l}\text { Zombie/ } \\
512\end{array}$ & [zJmbi:] \\
\hline & & ea & Eagle/132 & [i:gl] & Reach/343 & {$[\mathrm{ri}: \mathrm{t}]$} & Tea/424 & [ti:] \\
\hline & & & Ease/130 & [i:z] & Mean/253 & [mi:n] & Sea/370 & [si:] \\
\hline & & $\mathrm{e}$ & $\begin{array}{l}\text { Economic } \\
\text { s/368 }\end{array}$ & $\begin{array}{l}\text { [i:kənJm } \\
\text { Iks] }\end{array}$ & $\begin{array}{l}\text { Premium/ } \\
910\end{array}$ & $\begin{array}{l}\text { Pri:miə } \\
\mathrm{m}]\end{array}$ & - & - \\
\hline & & & Equal/388 & [i:kwəl] & $\begin{array}{l}\text { Demon/29 } \\
1\end{array}$ & $\begin{array}{l}\text { [di:mən } \\
\text { ] }\end{array}$ & - & - \\
\hline & & ae & $\begin{array}{l}\text { Aesthetic/ } \\
21\end{array}$ & [i:s $\theta \varepsilon t I k]$ & $\begin{array}{l}\text { Faeces/49 } \\
2\end{array}$ & [fi:si:z] & - & - \\
\hline & & & Aegis/21 & [i: dzIs] & - & - & - & - \\
\hline & & $\mathrm{i}$ & - & - & Libra/678 & [li:brə] & Ski/1108 & [ski:] \\
\hline & & & - & - & $\begin{array}{l}\text { Milieu/73 } \\
8\end{array}$ & [mi:ljə] & & \\
\hline & & $\mathrm{y}^{*}$ & & & $\begin{array}{l}\text { Embryo/4 } \\
49\end{array}$ & $\begin{array}{l}\text { [Embriə } \\
\text { u] }\end{array}$ & $\begin{array}{l}\text { Yucky/l6 } \\
66\end{array}$ & [j $\Lambda \mathrm{ki}]$ \\
\hline & & & & & $\begin{array}{l}\text { Bodyguar } \\
\text { d/134 }\end{array}$ & $\begin{array}{l}\text { [bJdiga } \\
\text { :d] }\end{array}$ & $\begin{array}{l}\text { Yummy/1 } \\
666\end{array}$ & [j $\mathrm{jmi}$ \\
\hline \multirow[t]{3}{*}{2} & \multirow{3}{*}{$\begin{array}{c}{[\mathrm{I}} \\
]\end{array}$} & $\mathrm{i}$ & Inside/217 & [Insaid] & Rich/356 & [rItt] & - & - \\
\hline & & & $\begin{array}{l}\text { Impress/2 } \\
07\end{array}$ & [Impres] & Skip/400 & [skIp] & - & - \\
\hline & & $\mathrm{e}$ & $\begin{array}{l}\text { Export/14 } \\
6\end{array}$ & $\begin{array}{l}\text { [Iksp3:(r } \\
) t]\end{array}$ & $\begin{array}{l}\text { Dessert/11 } \\
4\end{array}$ & $\begin{array}{l}\text { [dIso:(r } \\
) t]\end{array}$ & Acne/4 & [æknI] \\
\hline
\end{tabular}




\begin{tabular}{|c|c|c|c|c|c|c|c|c|}
\hline & & & Estate/141 & [Isteit] & Filter/153 & $\begin{array}{l}{[\mathrm{fIlt} \partial(\mathrm{r})} \\
]\end{array}$ & $\begin{array}{l}\text { Simile/3 } \\
85\end{array}$ & [simIlI] \\
\hline & & ie & - & - & - & - & $\begin{array}{l}\text { Talkie/1 } \\
220\end{array}$ & [tJ:lkI] \\
\hline & & & - & - & - & - & $\begin{array}{l}\text { Sortie/11 } \\
35\end{array}$ & [s?:rtI] \\
\hline & & ae & - & - & $\begin{array}{l}\text { Caesura/1 } \\
78\end{array}$ & $\begin{array}{l}\text { [sIzjəur } \\
\text { ə] }\end{array}$ & $\begin{array}{l}\text { Guinea/6 } \\
34\end{array}$ & [gIni] \\
\hline 3 & $\begin{array}{c}{[\varepsilon} \\
]\end{array}$ & $\mathrm{e}$ & $\begin{array}{l}\text { Ecstasy/13 } \\
1\end{array}$ & $\begin{array}{l}\text { [ Ekstəsi } \\
]\end{array}$ & $\begin{array}{l}\text { Election/1 } \\
33\end{array}$ & $\begin{array}{l}\text { [ } \\
\text { Ilek } \int ə n\end{array}$ & - & - \\
\hline & & & $\begin{array}{l}\text { Estimate/1 } \\
41\end{array}$ & [Estimeit] & Lend/238 & [ lend ] & - & - \\
\hline & & $\mathrm{a}$ & Any/48 & [عni] & Rare/1169 & [r\&r ] & - & - \\
\hline & & & $\begin{array}{l}\text { Anyplace/ } \\
49\end{array}$ & [Enipleis] & $\begin{array}{l}\text { February/5 } \\
09\end{array}$ & $\begin{array}{l}\text { [febjucr } \\
\text { i ] }\end{array}$ & - & - \\
\hline & & ea & - & - & Dead/107 & [ded] & - & - \\
\hline & & & - & - & Sweat/418 & [swet] & - & - \\
\hline 4 & $\begin{array}{c}{[\mathfrak{x}} \\
]\end{array}$ & $\mathrm{a}$ & $\begin{array}{l}\text { Ambition/ } \\
35\end{array}$ & [æbifən ] & Back/73 & [bæk ] & - & - \\
\hline & & & Absent/4 & [æbsənt ] & Lamp/233 & [læmp ] & - & - \\
\hline 5 & {$\left[\begin{array}{c}\partial \\
]\end{array}\right.$} & $\mathrm{a}$ & About/2 & [ obaut ] & $\begin{array}{l}\text { Fatigue/50 } \\
6\end{array}$ & $\begin{array}{l}\text { [ fət:ig } \\
\text { ] }\end{array}$ & $\begin{array}{l}\text { Puma/33 } \\
4\end{array}$ & [ pjumə ] \\
\hline & & & Above/3 & {$[\mathrm{\partial b} \Lambda \mathrm{v}]$} & $\begin{array}{l}\text { Nectar/27 } \\
6\end{array}$ & {$[\operatorname{nct\partial }(\mathrm{r})$} & $\begin{array}{l}\text { Umbrella } \\
/ 449\end{array}$ & $\begin{array}{l}{[\Lambda \mathrm{mbrcl} \text { }} \\
]\end{array}$ \\
\hline & & 0 & $\begin{array}{l}\text { Oblique/9 } \\
74\end{array}$ & [ obli:k ] & $\begin{array}{l}\text { Protect/93 } \\
2\end{array}$ & $\begin{array}{l}\text { [ protek } \\
\text { ] }\end{array}$ & - & - \\
\hline & & & $\begin{array}{l}\text { Object/28 } \\
2\end{array}$ & $\begin{array}{l}\text { [ abdz\&k } \\
\text { ] }\end{array}$ & $\begin{array}{l}\text { Common/ } \\
228\end{array}$ & $\begin{array}{l}{[} \\
\text { kJmən } \\
]\end{array}$ & - & - \\
\hline & & $\mathrm{e}$ & - & - & $\begin{array}{l}\text { Parent/102 } \\
8\end{array}$ & $\begin{array}{l}\text { [ } \\
\text { peərənt } \\
]\end{array}$ & - & - \\
\hline & & & - & - & $\begin{array}{l}\text { Energy/45 } \\
3\end{array}$ & $\begin{array}{l}\text { [enədzi } \\
\text { ] }\end{array}$ & - & - \\
\hline & & $\mathrm{u}$ & $\begin{array}{l}\text { Upon/131 } \\
3\end{array}$ & [ әpつn ] & Figure/433 & $\begin{array}{l}\text { [ fIgə(r) } \\
\text { ] }\end{array}$ & - & - \\
\hline & & & $\begin{array}{l}\text { Unless/15 } \\
73\end{array}$ & [ ənles ] & $\begin{array}{l}\text { Gerund/49 } \\
3\end{array}$ & $\begin{array}{l}\text { [dzerən } \\
\text { d ] }\end{array}$ & - & - \\
\hline
\end{tabular}




\begin{tabular}{|c|c|c|c|c|c|c|c|c|}
\hline & & $\mathrm{i}$ & - & - & Direct/378 & [dər\&k ] & - & - \\
\hline \multirow[t]{8}{*}{6} & \multirow{8}{*}{ [ə:] } & ir & - & - & Bird/117 & [bə:d] & - & - \\
\hline & & & - & - & Dirty/380 & [də:ti] & - & - \\
\hline & & ur & $\begin{array}{l}\text { Urgent/13 } \\
14\end{array}$ & [ə:dzənt] & Burn/150 & $\begin{array}{l}\text { [bə:(r)n } \\
\text { ] }\end{array}$ & - & - \\
\hline & & & Urge/1314 & [ə:d)] & Turn/1284 & [tə:(r)n] & - & - \\
\hline & & ear & Earth/364 & [ə:(r) $\theta]$ & Learn/671 & [lə:n] & - & - \\
\hline & & & Early/364 & [ə:(r)li] & Pearl/853 & [рә:1] & - & - \\
\hline & & er & Ergo/462 & [ə:gəu] & Assert/65 & $\begin{array}{l}\text { [əsə:(r)t } \\
\text { ] }\end{array}$ & - & - \\
\hline & & & Err/462 & [ə:(r)] & Astern/67 & $\begin{array}{l}\text { [əstə:(r) } \\
\mathrm{n}]\end{array}$ & - & - \\
\hline
\end{tabular}

The following is the table showing the correspondence between phonetic writing and orthographic writing for back vowels.

Tabel 3

Correspondence from Phonetic Transcription (Back Vowels) to the Orthographic Transcription (Alphabets)

\begin{tabular}{|c|c|c|c|c|c|c|c|c|}
\hline \multirow{3}{*}{ No } & \multirow{3}{*}{$\begin{array}{l}\tilde{n} \\
\tilde{E} \\
0 \\
\dot{0}\end{array}$} & \multirow{3}{*}{$\begin{array}{l}\frac{n}{0} \\
\frac{0}{\pi} \\
\frac{\pi}{2} \\
\frac{1}{4}\end{array}$} & \multicolumn{6}{|c|}{3.3 D I S T R I B U T I O N } \\
\hline & & & \multicolumn{2}{|c|}{ Initial Position } & \multicolumn{2}{|c|}{ Mid Position } & \multicolumn{2}{|c|}{ Final Position } \\
\hline & & & $\begin{array}{c}\text { Words and } \\
\text { page }\end{array}$ & $\begin{array}{l}\text { Phonetic } \\
\text { transcript } \\
\text { ion }\end{array}$ & $\begin{array}{c}\text { Words } \\
\text { and page }\end{array}$ & $\begin{array}{l}\text { Phonetic } \\
\text { transcript } \\
\text { ion }\end{array}$ & $\begin{array}{c}\text { Words } \\
\text { and page }\end{array}$ & $\begin{array}{c}\text { Phonetic } \\
\text { transcript } \\
\text { ion }\end{array}$ \\
\hline \multirow[t]{5}{*}{1} & \multirow[t]{5}{*}{ [u:] } & $\mathrm{O}$ & - & - & $\begin{array}{l}\text { Move/76 } \\
0\end{array}$ & [mu:v] & Do/342 & {$[\mathrm{du}:]$} \\
\hline & & & - & - & $\begin{array}{l}\text { Prove/93 } \\
3\end{array}$ & [pru:v] & Ado/16 & [ədu:] \\
\hline & & Oo & $\begin{array}{l}\text { Oodles/81 } \\
0\end{array}$ & [u:dlz] & Pool/320 & [pu:1] & $\begin{array}{l}\text { Zoo/139 } \\
2\end{array}$ & [zu:] \\
\hline & & & Ooze/810 & [u:z] & $\begin{array}{l}\text { Room/10 } \\
20\end{array}$ & [ru:m] & $\begin{array}{l}\text { Bamboo/ } \\
27\end{array}$ & $\begin{array}{l}\text { [bæmbu: } \\
\text { ] }\end{array}$ \\
\hline & & $\mathrm{Ou}$ & - & - & Route/12 & [ru:t] & - & - \\
\hline
\end{tabular}




\begin{tabular}{|c|c|c|c|c|c|c|c|c|}
\hline & & & & & 37 & & & \\
\hline & & & - & - & $\begin{array}{l}\text { Routine/ } \\
1237\end{array}$ & [ru:ti:n] & - & - \\
\hline \multirow[t]{6}{*}{2} & \multirow[t]{6}{*}{ [U] } & Oo & $\begin{array}{l}\text { Oomph/81 } \\
0\end{array}$ & [Umf] & $\begin{array}{l}\text { Wood/47 } \\
6\end{array}$ & [wUd] & & \\
\hline & & & - & - & Book/45 & [bUk] & - & - \\
\hline & & $\mathrm{Ou}$ & - & - & $\begin{array}{l}\text { Should/3 } \\
93\end{array}$ & {$\left[\int \mathrm{Ud}\right]$} & - & - \\
\hline & & & - & - & $\begin{array}{l}\text { Could/19 } \\
4\end{array}$ & [kUd] & - & - \\
\hline & & $\mathrm{U}$ & $\begin{array}{l}\text { Umlaut/15 } \\
62\end{array}$ & [Umlaut] & Bull/147 & [bUl] & - & - \\
\hline & & & - & - & Push/944 & {$\left[\mathrm{pU} \int\right]$} & - & - \\
\hline \multirow[t]{2}{*}{3} & \multirow[t]{2}{*}{ [כ] } & $\mathrm{O}$ & $\begin{array}{l}\text { Obvious/7 } \\
99\end{array}$ & [Jbvios] & $\begin{array}{l}\text { Loyal/24 } \\
4\end{array}$ & [1วjəl] & - & - \\
\hline & & & $\begin{array}{l}\text { Operation/ } \\
288\end{array}$ & $\begin{array}{l}\text { [Jpreifən } \\
\text { ] }\end{array}$ & $\begin{array}{l}\text { Knock/2 } \\
31\end{array}$ & [nכk] & - & - \\
\hline \multirow[t]{10}{*}{4} & \multirow[t]{10}{*}{$\begin{array}{c}\text { [כ: } \\
\text { ] }\end{array}$} & $\mathrm{A}$ & All/29 & [כ:1] & $\begin{array}{l}\text { Talk/122 } \\
0\end{array}$ & {$[\mathrm{t}$ J:k] } & - & - \\
\hline & & & Alter/12 & [Oltə(r)] & $\begin{array}{l}\text { Waitz/16 } \\
07\end{array}$ & [w]:Is] & - & - \\
\hline & & Or & Order/997 & [J:rdə(r)] & Corn/91 & [kJ:n] & - & - \\
\hline & & & Oral/997 & [J:rəl] & $\begin{array}{l}\text { Short/38 } \\
1\end{array}$ & [โว:t] & - & - \\
\hline & & $\begin{array}{c}\mathrm{Oa} \\
\mathrm{r}\end{array}$ & Oar/797 & {$[\mathrm{J}:(\mathrm{r})]$} & $\begin{array}{l}\text { Board/11 } \\
9\end{array}$ & {$[\mathrm{bJ}:(\mathrm{r}) \mathrm{d}]$} & - & - \\
\hline & & & $\begin{array}{l}\text { Oarsman/7 } \\
96\end{array}$ & [J:zmən] & $\begin{array}{l}\text { Coarse/2 } \\
14\end{array}$ & [kJ:s] & - & - \\
\hline & & $\mathrm{Ou}$ & $\begin{array}{l}\text { Ought/100 } \\
2\end{array}$ & {$[\mathrm{~J}: \mathrm{t}]$} & $\begin{array}{l}\text { Bought/1 } \\
44\end{array}$ & [bJ:t] & - & - \\
\hline & & & - & - & $\begin{array}{l}\text { Thought/ } \\
1504\end{array}$ & {$[\theta \supset: t]$} & - & - \\
\hline & & $\mathrm{Au}$ & $\begin{array}{l}\text { Audible/7 } \\
1\end{array}$ & [J:dIbəl] & $\begin{array}{l}\text { Taut/147 } \\
7\end{array}$ & [t]:t] & - & - \\
\hline & & & $\begin{array}{l}\text { Audience/ } \\
71\end{array}$ & [J:diəns] & $\begin{array}{l}\text { Taunt/14 } \\
77\end{array}$ & [t]: nt] & - & - \\
\hline \multirow[t]{2}{*}{5} & \multirow[t]{2}{*}{$\begin{array}{c}{[\Lambda} \\
]\end{array}$} & $\mathrm{Ou}$ & - & - & $\begin{array}{l}\text { Tough/1 } \\
264\end{array}$ & {$[\mathrm{t} \Lambda \mathrm{f}]$} & - & - \\
\hline & & & - & - & $\begin{array}{l}\text { Couple/2 } \\
66\end{array}$ & [ k $\wedge \mathrm{pl}]$ & - & - \\
\hline
\end{tabular}




\begin{tabular}{|c|c|c|c|c|c|c|c|c|}
\hline & & $\mathrm{O}$ & Onion/287 & [ $\Lambda$ nion ] & Come/76 & [ knm ] & - & - \\
\hline & & & $\begin{array}{l}\text { Otherwise/ } \\
291\end{array}$ & $\begin{array}{l}\text { [ } \\
\Lambda \text { ðəwaiz } \\
]\end{array}$ & $\begin{array}{l}\text { Month/7 } \\
53\end{array}$ & {$[\mathrm{~m} \wedge \mathrm{n} \theta]$} & - & - \\
\hline & & $\mathrm{U}$ & $\begin{array}{l}\text { Upset/131 } \\
3\end{array}$ & [ $\Lambda \mathrm{psct}]$ & Fun/480 & {$[\mathrm{f} \Lambda \mathrm{n}]$} & - & - \\
\hline & & & Utter/456 & {$[\Lambda \mathrm{t} \partial(\mathrm{r})]$} & $\begin{array}{l}\text { Plum/31 } \\
7\end{array}$ & [plım ] & - & - \\
\hline & & Oo & - & - & $\begin{array}{l}\text { Blood/11 } \\
5\end{array}$ & [blıd ] & - & - \\
\hline & & & - & - & $\begin{array}{l}\text { Flood/44 } \\
9\end{array}$ & [ fl $\Lambda \mathrm{d}]$ & & \\
\hline 6 & [a:] & $\mathrm{A}$ & Answer42 & [a:nsə(r)] & $\begin{array}{l}\text { Advance } \\
/ 17\end{array}$ & [ədva:ns] & $\mathrm{Bra} / 131$ & [bra:] \\
\hline & & & After/21 & [a:ftə(r)] & Fast/423 & [fa:st] & $\mathrm{Spa} / 1137$ & [spa:] \\
\hline & & $\mathrm{Ar}$ & Arbiter/51 & $\begin{array}{l}\text { [a:bitə(r) } \\
\text { ] }\end{array}$ & $\begin{array}{l}\text { Farm/42 } \\
1\end{array}$ & [fa:(r)m] & Far/420 & [fa:] \\
\hline & & & Arm/53 & [a:m] & $\begin{array}{l}\text { Charge/1 } \\
86\end{array}$ & [ta:(r)dz] & Car/166 & [ka:] \\
\hline
\end{tabular}

It can be seen in table 2 and table 3 that the vowels [ $\varepsilon$, [u:] and [U] correspond to the orthographic transcription in three forms, the vowels $[I],[ə:]$, and $[\Lambda]$ in four forms, the vowels [ə] and [ว:] in five forms, and the vowel [i:] in seven forms. It indicates that there is a complex correspondence between phonetic transcription and orthographic transcription in this respect. However, not all the forms of correspondence have complete distribution. Even, the forms with complete distribution are found less than the forms with partial distribution.

The vowel [i:], for example, corresponds to the alphabets $\boldsymbol{e} \boldsymbol{e}$ and $\boldsymbol{e a}$ with complete distribution, whereas the other alphabets $-\boldsymbol{i} \boldsymbol{e}, \boldsymbol{e}, \boldsymbol{a} \boldsymbol{e}, \boldsymbol{i}$, and $\boldsymbol{y}$ - have partial distribution. Then, the alphabet $\boldsymbol{e}$ only corresponds to the vowel [I] with complete distribution; meanwhile, the alphabets $\boldsymbol{i}$, ie, andaecan correspond to [I] with partial distribution. The distribution which is totally partial occurs to the vowel $[\varepsilon]$ which corresponds to the alphabets $\boldsymbol{e}, \boldsymbol{a}$, andea . Even, $[\varepsilon]$ only corresponds to the alphabets eain mid position.

The vowel [ə] corresponds to the alphabet $\boldsymbol{a}$ with complete distribution. The other alphabets, $\boldsymbol{o}$ and $\boldsymbol{u}$ can correspond to [ə] when occuring in initial and mid positions, andeandiin mid position only. However, the alphabet $i$ can correspond to [ə] in a limited case or context.

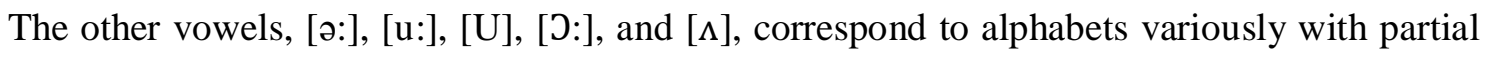


distribution, except the vowel [u:] which can correspond to the alphabets oo which can be found in all positions.

Similar phenomena happen to the diphthongs. Even, some forms of correspondence with diphthongs are very specific and limited. The diphthong [ai] corresponds to the alphabets $\boldsymbol{i} \boldsymbol{e}$ in mid position limitedly, and so does the sound [iə] for the alphabets $\boldsymbol{e e r}$ and $\boldsymbol{e}$ in initial position. For more complete picture of the correspondence between the phonetic transcription and the orthographic transcription for diphthongs, the following tables (table 4 and table 5) can be referred.

Tabel 4

Correspondence from Phonetic Transcription (Non-CenteringDiphthong) to the Orthographic Transcription (Alphabets)

\begin{tabular}{|c|c|c|c|c|c|c|c|c|}
\hline \multirow{3}{*}{ No } & \multirow{3}{*}{$\begin{array}{l}\tilde{z} \\
\vdots \\
\tilde{\sigma}\end{array}$} & \multirow{3}{*}{$\begin{array}{l}\frac{n}{0} \\
\frac{\pi}{\pi} \\
\frac{\pi}{2} \\
\frac{1}{4}\end{array}$} & \multicolumn{6}{|c|}{3.4 D I S T R I B U T I O N } \\
\hline & & & \multicolumn{2}{|c|}{ Initial Position } & \multicolumn{2}{|c|}{ Mid Position } & \multicolumn{2}{|c|}{ Final Position } \\
\hline & & & $\begin{array}{l}\text { Words } \\
\text { and page }\end{array}$ & $\begin{array}{c}\text { Phonetic } \\
\text { transcripti } \\
\text { on }\end{array}$ & $\begin{array}{c}\text { Words and } \\
\text { page }\end{array}$ & $\begin{array}{c}\text { Phonetic } \\
\text { transcripti } \\
\text { on }\end{array}$ & $\begin{array}{l}\text { Words } \\
\text { and page }\end{array}$ & $\begin{array}{l}\text { Phonetic } \\
\text { transcript } \\
\text { ion }\end{array}$ \\
\hline \multirow[t]{6}{*}{1} & \multirow[t]{6}{*}{ [ei] } & $\bar{a}$ & Ace/4 & [eis] & Blame/38 & [bleim] & - & - \\
\hline & & & Age/8 & [eids] & Game/171 & [geim] & - & - \\
\hline & & ai & $\operatorname{Aim} / 9$ & [eim] & Chain/62 & [tein] & - & - \\
\hline & & & $\begin{array}{l}\text { Ailment/ } \\
25\end{array}$ & [eilmənt] & Praise/323 & [preiz] & - & - \\
\hline & & ay & - & - & - & - & Say/1044 & [sei] \\
\hline & & & - & - & - & - & Pray/906 & [prei] \\
\hline \multirow[t]{6}{*}{2} & \multirow[t]{6}{*}{$\begin{array}{c}{[\partial u} \\
]\end{array}$} & $\mathrm{O}$ & $\begin{array}{l}\text { Ocean/2 } \\
84\end{array}$ & [əu]n] & Poster/322 & [pəustə(r)] & Go/179 & [gəu] \\
\hline & & & $\begin{array}{l}\text { Open/28 } \\
8\end{array}$ & [əupən] & Total/438 & [təutəl] & $\mathrm{No} / 279$ & [nəu] \\
\hline & & ow & Owe/295 & [วu] & Grown/196 & [grəun] & $\begin{array}{l}\text { Flow/16 } \\
0\end{array}$ & [fləu] \\
\hline & & & Own/295 & [oun] & Shown/382 & [Joun] & Low/248 & [ləu] \\
\hline & & oa & Oak/282 & [ouk] & Boat/41 & [bəut] & - & - \\
\hline & & & Oath/282 & [əu $\theta]$ & Loan/245 & [loun] & - & - \\
\hline \multirow[t]{2}{*}{3} & \multirow[t]{2}{*}{ [ai] } & $\mathrm{i}$ & Idea/588 & [aidiə] & Arise $/ 18$ & [əraiz] & Alibi/28 & [æləbai] \\
\hline & & & Ice/587 & [ais] & Child/192 & [tgaild] & Alkali/29 & [ælkəlai] \\
\hline
\end{tabular}




\begin{tabular}{|c|c|c|c|c|c|c|c|c|}
\hline & & \multirow[t]{2}{*}{ ie } & - & - & $\begin{array}{l}\text { Hierarchy/6 } \\
72\end{array}$ & [haira:ki] & Die/116 & [dai] \\
\hline & & & - & - & Diet/114 & [daiət] & Tie/434 & [tai] \\
\hline & & uy & - & - & - & - & Buy/53 & [bai] \\
\hline & & & - & - & - & - & Guy/187 & [gai] \\
\hline & & $\mathrm{Y}$ & - & - & Type/471 & [taip] & - & - \\
\hline & & & - & - & $\begin{array}{l}\text { Dynamic/12 } \\
9\end{array}$ & $\begin{array}{l}\text { [dainæmIk } \\
\text { ] }\end{array}$ & - & - \\
\hline \multirow[t]{4}{*}{4} & \multirow[t]{4}{*}{$\begin{array}{c}{[\mathrm{au}} \\
]\end{array}$} & ou & $\begin{array}{l}\text { Output/1 } \\
006\end{array}$ & [autpUt] & Bound/43 & [baund] & - & - \\
\hline & & & $\begin{array}{l}\text { Ounce/2 } \\
91\end{array}$ & [auns] & Mouse/760 & [maus] & - & - \\
\hline & & ow & Ow/829 & [au] & Brown/49 & [braun] & Bow/44 & [bau] \\
\hline & & & Owl/830 & [aul] & Town/439 & [taun] & Now/281 & [nau] \\
\hline \multirow[t]{4}{*}{5} & \multirow[t]{4}{*}{$\begin{array}{c}{[\mathrm{Ji}} \\
]\end{array}$} & oi & Oil/805 & [Jil] & Boil/41 & [bJil] & $\begin{array}{l}\text { Hoipollo } \\
\text { i/566 }\end{array}$ & $\begin{array}{l}\text { [hJipəlJi } \\
\text { ] }\end{array}$ \\
\hline & & & $\begin{array}{l}\text { Oilcloth/ } \\
805\end{array}$ & {$[$ JilklJ $\theta]$} & Poison/319 & [pJizn] & - & - \\
\hline & & oy & $\begin{array}{l}\text { Oyster/8 } \\
30\end{array}$ & {$[$ Jistə(r)] } & Coyote/314 & [kJi əut] & $\begin{array}{l}\text { Annoy/1 } \\
4\end{array}$ & [ənJi] \\
\hline & & & - & - & $\begin{array}{l}\text { Voyage/160 } \\
2\end{array}$ & [vJi Ids] & $\begin{array}{l}\text { Enjoy/13 } \\
8\end{array}$ & [Ind3Ji] \\
\hline
\end{tabular}

The following is the table showing the correspondence between phonetic writing and orthographic writing for centering diphthogs.

Tabel 5

Correspondence from Phonetic Transcription (CenteringDiphthong) to the Orthographic Transcription (Alphabets)

\begin{tabular}{|c|c|c|c|c|c|c|c|c|}
\hline \multirow{3}{*}{ No } & \multirow{3}{*}{ 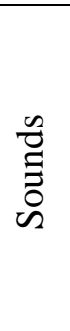 } & \multirow{3}{*}{$\begin{array}{l}\frac{n}{0} \\
\frac{0}{\pi} \\
\frac{\pi}{2} \\
\frac{2}{2}\end{array}$} & \multicolumn{6}{|c|}{$3.5 \quad$ D I S T R I B U T I O N } \\
\hline & & & \multicolumn{2}{|c|}{ Initial Position } & \multicolumn{2}{|c|}{ Mid Position } & \multicolumn{2}{|c|}{ Final Position } \\
\hline & & & $\begin{array}{c}\text { Words } \\
\text { and page }\end{array}$ & $\begin{array}{c}\text { Phonetic } \\
\text { transcript } \\
\text { ion }\end{array}$ & $\begin{array}{c}\text { Words } \\
\text { and page }\end{array}$ & $\begin{array}{l}\text { Phonetic } \\
\text { transcript } \\
\text { ion }\end{array}$ & $\begin{array}{c}\text { Words } \\
\text { and page }\end{array}$ & $\begin{array}{c}\text { Phonetic } \\
\text { transcripti } \\
\text { on }\end{array}$ \\
\hline \multirow[t]{2}{*}{1} & \multirow[t]{2}{*}{$\begin{array}{c}\text { [ } \\
\text { Iə] }\end{array}$} & ea & Ear/132 & [ Iə] & Dear/107 & [ dIə] & $\begin{array}{l}\text { Cornea/2 } \\
59\end{array}$ & [kJnIə] \\
\hline & & & $\begin{array}{l}\text { Earring/3 } \\
64\end{array}$ & 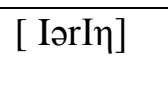 & Near/193 & [ nIə] & - & - \\
\hline
\end{tabular}




\begin{tabular}{|c|c|c|c|c|c|c|c|c|}
\hline & & eer & $\begin{array}{l}\text { Eerie/36 } \\
9\end{array}$ & [ Iəri] & - & - & $\begin{array}{l}\text { Cheer/19 } \\
0\end{array}$ & [t'Iə] \\
\hline & & & & & - & - & $\begin{array}{l}\text { Steer/40 } \\
6\end{array}$ & [stIə] \\
\hline & & ior & - & - & - & - & $\begin{array}{l}\text { Inferior/ } \\
609\end{array}$ & [ InfiərIə] \\
\hline & & & - & - & - & - & $\begin{array}{l}\text { Interior/6 } \\
23\end{array}$ & [ IntiərIə] \\
\hline & & $\mathrm{e}$ & Era/461 & [ Iərə] & - & - & - & - \\
\hline & & & & & - & - & - & - \\
\hline & & ia & - & - & - & - & $\begin{array}{l}\text { Bacteria/ } \\
81\end{array}$ & $\begin{array}{l}\text { [bæktIərIə } \\
\text { ] }\end{array}$ \\
\hline & & & - & - & - & - & $\begin{array}{l}\text { Criteria/ } \\
322\end{array}$ & [kraitIərIə] \\
\hline 2 & $\begin{array}{c}{[\varepsilon \partial} \\
]\end{array}$ & are & - & - & - & - & $\begin{array}{l}\text { Stare/11 } \\
63\end{array}$ & [steə] \\
\hline & & & - & - & - & - & $\begin{array}{l}\text { Share/37 } \\
8\end{array}$ & {$\left[\int \varepsilon \partial\right]$} \\
\hline & & air & Air/9 & [عə] & - & - & Stair/404 & [steə] \\
\hline & & & Airy/10 & [Eəri] & - & - & Hair/195 & [hEə] \\
\hline & & ear & - & - & - & - & $\begin{array}{l}\text { Wear//46 } \\
7\end{array}$ & [w\&ə] \\
\hline & & & - & - & - & - & $\begin{array}{l}\text { Swear/14 } \\
58\end{array}$ & [sw\&ə] \\
\hline & & ae & Aerial/21 & [Eərial] & - & - & - & - \\
\hline & & & $\begin{array}{l}\text { Aerobics } \\
/ 21\end{array}$ & $\begin{array}{l}\text { [Eərəubik } \\
\text { s] }\end{array}$ & - & - & - & - \\
\hline 3 & $\begin{array}{c}\text { [uə } \\
\text { ] }\end{array}$ & ur & - & - & $\begin{array}{l}\text { Curious/ } \\
103\end{array}$ & $\begin{array}{l}\text { [kjuoriəs } \\
\text { ] }\end{array}$ & - & - \\
\hline & & & - & - & $\begin{array}{l}\text { Purify/33 } \\
5\end{array}$ & $\begin{array}{l}\text { [pjuorifai } \\
\text { ] }\end{array}$ & - & - \\
\hline & & ua & - & - & $\begin{array}{l}\text { Mutual/2 } \\
73\end{array}$ & $\begin{array}{l}\text { [mju:tyo } \\
\text { 1] }\end{array}$ & - & - \\
\hline & & & - & - & $\begin{array}{l}\text { Sanctuar } \\
\text { y / } 365\end{array}$ & $\begin{array}{l}\text { [sæクtfuər } \\
\text { i] }\end{array}$ & - & - \\
\hline & & oor & - & - & - & - & $\begin{array}{l}\text { moor/92 } \\
3\end{array}$ & [muə] \\
\hline & & & & & - & - & Boor/139 & [buə] \\
\hline & & ure & - & - & - & - & Cure/286 & [kjuə(r)] \\
\hline
\end{tabular}




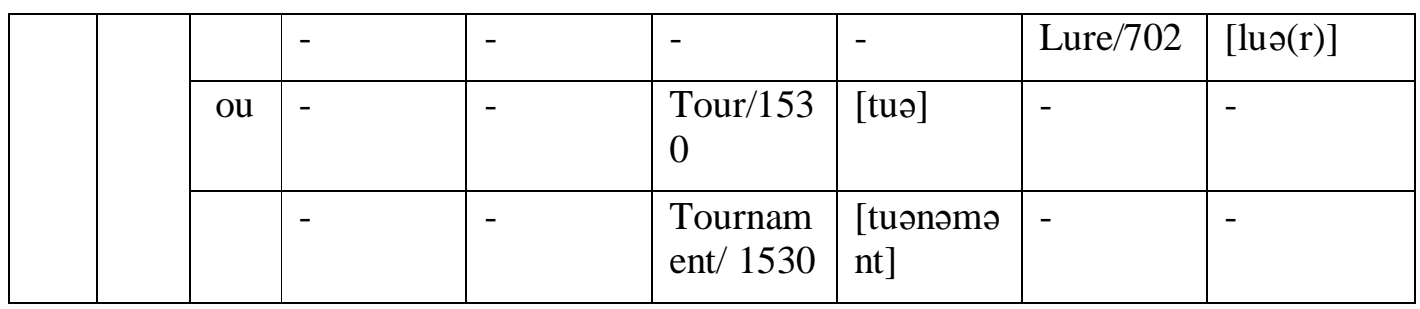

2. The most Complex and Simplest English Phonetic Symbols (Transcription)

This part is used to discuss the most complex and simplest English phonetic symbols. The complexity and simplicity of the phonetic symbols can only be understood when they are already used in words corresponding to the orthographic transcription (alphabets). Therefore, what is meant by the most complex phonetic symbol here is the sound (phonetic) symbol (transcription) which corresponds to the orthographic transcription (alphabets) having the most various forms. In contrast, when the correspondence shows least variations or it shows one-to-one correspondence between the phonetic symbol and the orthographic symbol, it means it is the simplest correspondence.

Meanwhile, the phonetic (vowel) symbol which has the most complex variations forms) is [i:] which corresponds to eight different forms of alphabets (orhographic transcription), namely $\boldsymbol{e} \boldsymbol{e}, \boldsymbol{i} \boldsymbol{e}, \boldsymbol{e a}, \boldsymbol{e}, \boldsymbol{a e}, \boldsymbol{i}$ and $\boldsymbol{y}$. The other phonetic symbols have less than eight forms of alphabets. Then, in this category, the simplest phonetic (vowel) symbol is [æ] which has only one form of alphabets, namely $\boldsymbol{a}$.To make it clearer, the following table can be viewed as a reference.

Table 6

Summary of the Correspondence between Phonetic Transcription and Orthographic Transcription Seen from Phonetic Transcription (Vowels)

\begin{tabular}{|c|c|c|c|c|c|c|c|c|c|c|}
\hline \multirow{2}{*}{ No } & \multirow{2}{*}{$\begin{array}{c}\text { Phonetic } \\
\text { Transcription } \\
\text { (Vowels) }\end{array}$} & \multicolumn{9}{|c|}{ Orthographic Transcription (Alphabets) } \\
\hline & & 1 & 2 & 3 & 4 & 5 & 6 & 7 & 8 & 9 \\
\hline 1 & [i:] & ee & ie & ea & $\mathrm{E}$ & ae & $\mathrm{i}$ & $\mathrm{y}$ & ei & \\
\hline 2 & {$[\mathrm{I}]$} & $\mathrm{i}$ & $\mathrm{e}$ & Ie & $\mathrm{Ae}$ & & & & & \\
\hline 3 & {$[\varepsilon]$} & $\mathrm{e}$ & $\mathrm{a}$ & $\mathrm{Ea}$ & & & & & & \\
\hline 4 & [æ] & $\mathrm{a}$ & & & & & & & & \\
\hline 5 & [ə] & $\mathrm{a}$ & o & $\mathrm{E}$ & $\mathrm{U}$ & $\mathrm{i}$ & & & & \\
\hline 6 & [ə:] & ir & ur & ear & Er & & & & & \\
\hline 7 & [u:] & $\mathrm{O}$ & OO & ou & & & & & & \\
\hline
\end{tabular}




\begin{tabular}{|c|c|c|c|c|c|c|c|c|c|c|}
\hline 8 & {$[\mathrm{U}]$} & oo & ou & $\mathrm{u}$ & & & & & & \\
\hline 9 & {$[\mathrm{~J}]$} & $\mathrm{o}$ & & & & & & & & \\
\hline 10 & {$[\mathrm{~J}:]$} & $\mathrm{a}$ & or & oar & Ou & au & & & & \\
\hline 11 & {$[\Lambda]$} & ou & o & $\mathrm{u}$ & Oo & & & & & \\
\hline 12 & {$[\mathrm{a}:]$} & $\mathrm{a}$ & $\mathrm{ar}$ & & & & & & & \\
\hline
\end{tabular}

In another case, the most complex phonetic symbols referring to diphthongs are [iə] and [uə] each of which corresponds to five different alphabets. The diphthong [iə] corresponds to the alphabets $\boldsymbol{e a}, \boldsymbol{e e r}$, ior, $\boldsymbol{e}$, andia, while the diphthong [uə] corresponds to the alphabets ur, ua, oor, ure, andou. Then, the simplest phonetic symbols referring to diphthong in this category are [au] and [Ji] which only correspond to two (groups of) alphabets, namely $\boldsymbol{o u}$ andow for [au] and $\boldsymbol{o i}$ and $\boldsymbol{o y}$ for [Ji]. To get a complete understanding related to this problem, the following table can be used a reference.

Table 7

Summary of the Correspondence between Phonetic Transcription and Orthographic Transcription Seen from Phonetic Transcription (Diphthongs)

\begin{tabular}{|c|c|c|c|c|c|c|c|c|c|c|}
\hline \multirow{2}{*}{ No } & $\begin{array}{c}\text { Phonetic } \\
\text { Transcription } \\
\text { (Diphthongs) }\end{array}$ & \multicolumn{8}{|c|}{ Orthographic Transcription (Alphabets) } \\
\hline & 1 & 2 & 3 & 4 & 5 & 6 & 7 & 8 & 9 \\
\hline 1 & {$[\mathrm{ei}]$} & a & ai & ay & & & & & & \\
\hline 2 & {$[$ [u] } & o & ow & oa & & & & & & \\
\hline 3 & {$[$ ai] } & i & ie & uy & Y & & & & & \\
\hline 4 & {$[\mathrm{au}]$} & ou & ow & & & & & & & \\
\hline 5 & {$[$ Ji] } & oi & oy & & & & & & & \\
\hline 6 & {$[$ [iə] } & ea & eer & ior & E & ia & & & & \\
\hline 7 & {$[\varepsilon ə]$} & are & air & ear & Ae & & & & & \\
\hline 8 & {$[\mathrm{u}]$} & ur & ua & oor & Ure & ou & & & & \\
\hline
\end{tabular}

3. The most Complex and Simplest English Orthographic Symbols (Alphabets Indicating Vowels)

Table 8 below can be used to know the most complex and simplest English orthographic symbol (alphabet indicating vowel). It shows that the most complex orthographic symbols indicating vowels are $\boldsymbol{o}$ followed by $\boldsymbol{a}$ which correspond respectively to nine and six different phonetic symbols. The alphabet $\boldsymbol{o}$ corresponds to the phonetic symbols

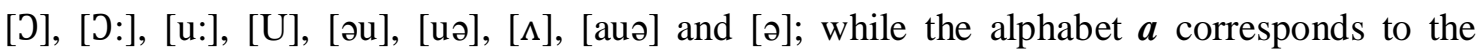


phonetic symbols [æ], [a:], [J:], [ə], [ع], and [ei]. Then, the simplest English alphabet

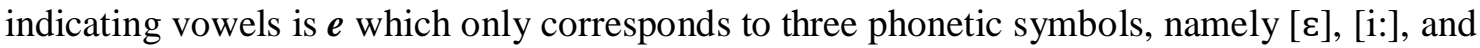
[iə].

Tabel 8

Summary of the Correspondence between Phonetic Transcription and Orthographic

Transcription Seen from Orthographic Transcription (Alphabets Indicating Vowels)

\begin{tabular}{|c|c|c|c|c|c|c|c|c|c|c|}
\hline \multirow{2}{*}{ No } & \multirow{2}{*}{$\begin{array}{c}\text { Orthographic } \\
\text { Transcription } \\
\text { (Alphabets) }\end{array}$} & \multicolumn{9}{|c|}{ Phonetic Transcription (Vowels and Diphthongs) } \\
\hline & & 1 & 2 & 3 & 4 & 5 & 6 & 7 & 8 & 9 \\
\hline 01 & $\mathrm{~A}$ & [æ] & [a:] & [כ:] & [ə] & {$[\varepsilon]$} & [ei] & & & \\
\hline 02 & $\mathrm{E}$ & {$[\varepsilon]$} & [i: & [iə] & & & & & & \\
\hline 03 & I & {$[\mathrm{I}]$} & [i:] & [ə:] & [ai] & & & & & \\
\hline 04 & $\mathrm{O}$ & [כ] & [כ:] & [u:] & [U] & [əu] & [uə] & {$[\Lambda]$} & [auə] & [ə] \\
\hline 05 & $\mathrm{U}$ & [U] & [u:] & {$[\Lambda]$} & [ə:] & [ju:] & & & & \\
\hline
\end{tabular}

4. CONCLUSION

Based on the previous discussion, some conclusions can be drawn as follows:

1. In general, the phonetic transcription (symbols) and the orthographic transcription (symbols) show one-to-many correspondence, although few of them show one-to-one correspondence.

2. Viewed from the phonetic transcription, one-to-one correspondence occurs in the vowels [æ] and [J], while for diphthongs, it does not occur.

3. Viewed from the orthographic transcription, there is no one-to-one correspondence between the orthographic symbols and the phonetic symbols.

4. The most complex phonetic symbol referring to vowels in English is the vowel [i:], while the simplest one is the vowel [æ].

5. The most complex phonetic symbols referring to diphthongs in English are the diphthongs [iə] and [uə], while the simplest one is the dipthong [au].

6. The most complex orthographic symbols (alphabets indicating vowels) are $\boldsymbol{o}$ followed by $\boldsymbol{a}$, while the simplest one is the alphabet $\boldsymbol{e}$. 


\section{REFERENCES}

Chaer, Abdul. (1994). Linguistik Umum. Jakarta: Rineka Cipta.

Djajasudarma, T Fatimah.(1993). Metode Linguistik : Ancangan Metode Penelitian and Kajian. Bandung: PT Eresco.

Hornby, A.S. (1986). Oxford Advanced Learner's Dictionary of Current English. Oxford: Oxford University Press.

Hyman, Larry M. (1975). Phonology: Theory and Analysis. New York: Holt, Rinehart and Winston Inc.

Longman, (2001).Longman Dictionary of Contemporary English. Edinburgh: Pearson Education Limited.

McMahon, April. (2002). An Introduction to English Phonology. Edinburgh: Edinburgh University Press.

Ramelan.(1985). English Phonetics. Semarang: IKIP Semarang Press.

Seliger, Herbert W and Elana Shohamy.(1989). SecondLanguage Research Methods. Oxford: Oxford University Press.

Singleton, Royce A. and Bruce C. Straits.(1999). Approaches to Social Research. Oxford: Oxford University Press.

Sloat, Clarence, Sharon Henderson Taylor, and James E. Hoard.(1978). Introduction to Phonology. New Jersey: Prentice-Hall, Inc. Englewood Cliffs.

Sudaryanto.(1993). Metode and Aneka Teknik Analisis Bahasa. Yogyakarta: Duta Wacana University Press. . (1986). Metode Linguistik. Yogyakarta: Gajah Mada University Press.

Verhaar, J.W.M. (1996). Asas-Asas Linguistik. Yogyakarta: Gadjah Mada University Press.

Widdoson, G. H. (1995). Principle and Practice in Applied Linguistics.Oxford: Oxford University Press. 